\title{
POLYMORPHISM OF MICROSATELLITE SEQUENCE WITHIN PROTEIN KINASE ORFS IN PHYTOPATHOGENIC FUNGUS, MAGNAPORTHE GRISEA
}

Chengyun $\mathrm{Li}^{1, *}$, Lin $\mathrm{Liu}^{1}$, Jing Yang ${ }^{1}$, Jinbin $\mathrm{Li}^{2}$, Zhang Yue ${ }^{1}$, Yunyue Wang ${ }^{1}$, Yong Xie ${ }^{1}$, Youyong Zhu ${ }^{1}$

${ }^{1}$ Key Laboratory for Agricultural Biodiversity and Pest Management of China Education Ministry, Plant Protection College, Yunnan Agricultural University, Kunming, 650201, China

2 Plant Protection Research Institute of Yunnan Academy of Agricultural Sciences, Kunming, 650205, China

* Correspondence: Chengyun Li, Key Laboratory for Agricultural Biodiversity and Pest Management of China Education Ministry, Yunnan Agricultural University, Hei Longtan, Kunming, 650201, China, Fax: 86-871-5227945,Email: li.chengyun@gmail.com

Abstract: Eighteen polymorphic microsatellite markers suitable for population genetic studies and protein kinase encoding genic variation measurement were developed for rice blast fungus Magnaporthe grisea. Polymorphism was evaluated by using 46 isolates collected from diverse geographical locations and rice varieties. Preliminary results indicate that each locus harbors two to fourteen alleles.

Keywords: Magnaporthe grisea; protein kinase; microsatellite

\section{INTRODUCTION}

Magnaporthe grisea is the most destructive pathogen of rice worldwide and the primary model organism for elucidating the molecular basis of fungal diseases of plants (Valent, 1990) The completion of the genome 
sequence for Magnaporthe grisea has made it possible to determine the total number of genes as well as to analyze and classify them according to their structure and function (Dean et al., 2005).

The eukaryotic protein kinases comprise one of the largest superfamilies of homologous proteins and genes. There are now hundreds of different members whose sequences are known within this family (Hanks, 2003). Although there are common structural features among protein kinases, differences in structural features, regulation modes, and substrate specificities divide them into separate groups. In the phytopathogenic fungi, components of heterotrimeric G proteins, MAP kinases, and cAMP signal transduction pathway are required for pathogenesis (Muller et al., 2003; Yamada-Okabe et al., 1999; Xu et al., 1996). We previous revealed that many protein kinase genes harbored SSR sequences within their protein coding region (Li C.Y. et al., 2005), but whether these sequences are polymorphic is unclear.

Microsatellites are favored for genetic applications because they are abundant in plant genomes, highly polymorphic within species, relatively rapid and inexpensive to assay, and can be used to identify specific chromosomal regions consistently across populations. Distribution and frequency of SSRs in genomic scale or ESTs have been analyzed extensively, however, reports published to date clearly discussed SSR polymorphism in genes has been limited (Li C.Y. et al., 2005).

The objective of this study was to develop PCR primer pairs targeting previously sequenced genes from $M$. grisea in order to compare the allelic amplification product polymorphism of protein kinase encoding genes among natural populations.

\section{MATERIAL AND METHOD}

The DNA sequence and a database of known and predicted open reading frames of eukaryotic protein kinases were obtained from the Magnaporthe grisea genome database (http://www.genome.wi.mit.edu/annotation/fungi/ magnaporthe/) on July 14, 2005. The program software tandem repeats finder (TRF) written by Benson (Benson, 1999) with the following options: minimum size $=15 \mathrm{bp}, 80 \%$ matches (namely number of matched bases between two repetitive elements is $80 \%$ ) and abundance were removed.

Polymorphic loci were detected by screening a subset of $46 \mathrm{M}$. grisea isolates collected from different locations and from various rice varieties of Yunnan Province, China. Genomic DNA was extracted from mycelia using a 
simple extraction protocol (Zhang et al., 1996). Primers were designed for DNA sequence with microsatellite motifs using PRIMER3 (Rozen et al., 2000) software and synthesized by Invitrongen Biotechnology Co. Ltd. Shanghai, China.

PCR amplifications were carried out in $20 \mu \mathrm{L}$ volumes containing $1 \times$ PCR buffer $\left(10 \mathrm{mM}\right.$ Tris- $\mathrm{HCl} \mathrm{pH} 8.5,50 \mathrm{mM} \mathrm{KCl}, 1.5 \mathrm{mM} \mathrm{MgCl}_{2}$, and $0.001 \%$ gelatin), $125 \mu \mathrm{M}$ each dNTP, 5 pmol of each primer, and $0.5 \mathrm{U}$ of Taq DNA polymerase (Sino-American Biotechnology Co., Beijing). Approximately $50 \mathrm{ng}$ of genomic DNA was used for each reaction. Amplification were performed in a Eppendoff PCR thermal Matercycler with the cycling parameters; $5 \mathrm{~min}$ at $94^{\circ} \mathrm{C}, 35$ cycles of $1 \mathrm{~min}$ at $94^{\circ} \mathrm{C}$, $1 \mathrm{~min}$ at $54^{\circ} \mathrm{C}$ and $1 \mathrm{~min}$ at $72^{\circ} \mathrm{C}$ followed by a final extension for $10 \mathrm{~min}$ at $72^{\circ} \mathrm{C}$. In initial experiments, amplified fragments were visualized by electrophoreses in $1.5 \%$ agarose gels stained with ethidium bromide. Loci that appeared polymorphic were further examined by $8 \%$ polyacrylamide gel to determine the product size of the PCR product and number of alleles per locus. Fragment size of PCR products were estimated on Bio-Imaging System E5000.

\section{RESULTS}

Eighteen of the 26 polymorphic loci produced amplicons from a majority of 46 isolates, and displayed anywhere from two to fourteen alleles (Table 1). Gene diversity was estimated with the software program, GENEPOP (V1.32), and are shown in table 1. KMS02, KMS07, KMS18 showed high gene diversity in population used for the study. This suggests that genes harboring these SSR sequences are also highly diverse within the populations.

M. grisea has 11109 proteins coding ORFs in whole genome, and among these, 85 protein kinase genes, corresponding to $-0.76 \%$ of the total number of genes (Dean et al., 2005). More than 30\% of these protein kinase encoding genes have SSRs within their protein coding regions. The high degree of polymorphism in this set of microsatellite markers can be used to analyze population structure and strain distribution in association with rice variety and location, adding to the fundamental understanding the function of protein kinase genes of the fungus. These results provide useful information to study possible SSR functions and variation of protein kinases that harbor them. 
Table 1. Polymorphsims of SSRs in protein kinase encoding ORFs in M. grisea. $\mathrm{N}_{\mathrm{a}}$, number of alleles; $\mathrm{G}_{\mathrm{d}}$ : Gene diversity by Shannon's Information inde

\begin{tabular}{|c|c|c|c|c|c|c|c|c|c|c|}
\hline Locus Contigt & PYIIIOS (F, $\left.5^{\prime}-3^{\prime \prime}\right)$ & Frimer $\left(R, S^{\prime}-3^{\prime}\right)$ & $\begin{array}{l}\text { Oene } \\
\text { name }\end{array}$ & Motir & $\begin{array}{l}\text { No of } \\
\text { Genotypes }\end{array}$ & $\begin{array}{c}\text { Reyps: } \\
\text { No. }\end{array}$ & $\mathrm{Al}$ & $\begin{array}{l}\text { Pavent } \\
\text { matches }\end{array}$ & $\begin{array}{l}\text { Pruduct sive } \\
\text { range (bp) }\end{array}$ & Gd \\
\hline KNSO12.1190 & $\begin{array}{l}\text { AGCGAAACAA } \\
\text { GAACGCGAGG }\end{array}$ & $\begin{array}{l}\text { TAGCAGCCTG } \\
\text { TGCTCGTTCG }\end{array}$ & $\begin{array}{l}\text { NGG } \\
064135\end{array}$ & $A O G$ & 46 & 5 & 2 & 100 & $219-222$ & 0.5623 \\
\hline KNSO22.1299 & $\begin{array}{l}\text { CGCAAAGAAT } \\
\text { TCAAAECCGC }\end{array}$ & $\begin{array}{l}\text { GGAGACGACA } \\
\text { CTGGGGTGCT }\end{array}$ & $\begin{array}{l}\text { NGGG } \\
070035\end{array}$ & $A D G$ & 46 & 6 & 14 & 100 & $279-326$ & 2.4256 \\
\hline KMSO3 2.1302 & $\begin{array}{l}\text { TCCCTTTCGGT } \\
\text { CGTTCCAAG }\end{array}$ & $\begin{array}{l}\text { CCCGCTGAGG } \\
\text { TAGCCAAAGA }\end{array}$ & $\begin{array}{l}\mathrm{NGG} \\
07012.5\end{array}$ & $A O G$ & 46 & 5 & 4 & 86 & $208-220$ & 1.0673 \\
\hline KMSO4 2.1631 & $\begin{array}{l}\text { CCGAAIGAGGT } \\
\text { CCTCCAAGCA }\end{array}$ & $\begin{array}{l}\text { GCAGCAGCAT } \\
\text { TACCATCCCC }\end{array}$ & $\begin{array}{l}\text { NGG } \\
086435\end{array}$ & AG & 46 & 6 & 5 & 88 & $216-311$ & 0.654 \\
\hline KMSS052.377 & $\begin{array}{l}\text { CCAAGCCCAG } \\
\text { AGCCAGAAAA }\end{array}$ & $\begin{array}{l}\text { CTCGAGGCTG } \\
\text { CCCATCTTGT }\end{array}$ & $\begin{array}{l}\text { NGG } \\
019985\end{array}$ & AGA & 46 & 5 & 3 & 85 & $243-249$ & 0.2826 \\
\hline KMSO62.1692 & $\begin{array}{l}\text { GCATGAAATG } \\
\text { CTCGTCGTGG }\end{array}$ & $\begin{array}{l}\text { ATGCAGCGGC } \\
\text { CTTCATTAGC }\end{array}$ & $\begin{array}{l}\text { NGG } \\
090005\end{array}$ & AGARAA & 46 & 3 & 4 & 100 & $191-209$ & 1.181 \\
\hline $\mathrm{KACS} 072.347$ & $\begin{array}{l}\text { GTTCTCCATCG } \\
\text { COCAAATCO }\end{array}$ & $\begin{array}{l}\text { TAAGTGGGCT } \\
\text { COTTOOCTOC }\end{array}$ & $\begin{array}{l}\text { NGG } \\
01816.5\end{array}$ & $\cos$ & 46 & 5 & 10 & 86 & $245-281$ & 2.1018 \\
\hline KNSOO 2993 & $\begin{array}{l}\text { GGTCCAATTCC } \\
\text { CCTTCOCCC }\end{array}$ & $\begin{array}{l}\text { TTCAACCCGAT } \\
\text { ACGAGGCCGT }\end{array}$ & $\begin{array}{l}\text { NGG } \\
053975\end{array}$ & CHAGG & 46 & 3 & 2 & 90 & $200-206$ & 0.5921 \\
\hline KNS112.211 & $\begin{array}{l}\text { GGGGTAACGA } \\
\text { CAGCCAGGTG }\end{array}$ & $\begin{array}{l}\text { CTGCTGCTGCT } \\
\text { GTTGCTGGT }\end{array}$ & $\begin{array}{l}\text { NGGG } \\
011965\end{array}$ & $\operatorname{CAG}$ & 46 & 7 & 5 & 80 & $218-230$ & 1.1916 \\
\hline KNS122.1645 & $\begin{array}{l}\text { CGATACCCCT } \\
\text { GAGCCACCAC }\end{array}$ & $\begin{array}{l}\text { ATCTGCCGCCT } \\
\text { TTTGAGTGC }\end{array}$ & $\begin{array}{l}\text { NGGOS } \\
46.5\end{array}$ & $\operatorname{CAg}$ & 46 & 5 & 6 & 100 & $198-213$ & 13926 \\
\hline KMS132.1353 & $\begin{array}{l}\text { GTCATGACAG } \\
\text { GGGTOTTCGG }\end{array}$ & $\begin{array}{l}\text { ATCCCCCTCTC } \\
\text { CCTCCATTC }\end{array}$ & $\begin{array}{l}\text { NGG } \\
072915\end{array}$ & $\operatorname{crgcoC}$ & 46 & 3 & 8 & 84 & $232-292$ & 1.8262 \\
\hline KMS14 2.1182 & $\begin{array}{l}\text { GAACCICGCA } \\
\text { GTCCAACAAC }\end{array}$ & $\begin{array}{l}\text { CGCCTGCTGA } \\
\text { GAATGGGACT }\end{array}$ & $\begin{array}{l}\text { NGG } \\
06368.5\end{array}$ & CAGTCA & 46 & 3 & 5 & 92 & $205-229$ & 12688 \\
\hline KNS152.838 & $\begin{array}{l}\text { GGCCALAGAG } \\
\text { GAGAACGGAA }\end{array}$ & $\begin{array}{l}\text { TCCATCAGGA } \\
\text { TCGGGGACTG }\end{array}$ & $\begin{array}{l}\text { NGG } \\
044635\end{array}$ & $O G A$ & 46 & 5 & 6 & 83 & $215-233$ & 1.7452 \\
\hline KMS162.343 & $\begin{array}{l}\text { TCATGAGCGA } \\
\text { GACAATGGGG }\end{array}$ & $\begin{array}{l}\text { TGCTGAACCG } \\
\text { ATTCCGCTTT }\end{array}$ & $\begin{array}{l}\text { NGGG } \\
01795.5\end{array}$ & OGGT & 46 & 4 & 2 & 83 & $206-210$ & 0.5623 \\
\hline KNS182.687 & $\begin{array}{l}\text { GCAAGICGCC } \\
\text { TCGCATTAT }\end{array}$ & $\begin{array}{l}\text { TGAACCGACT } \\
\text { CGTCGACTGC }\end{array}$ & $\begin{array}{l}\text { NGG } \\
03488.5\end{array}$ & $\mathrm{GCA}$ & 46 & 6 & 5 & 82 & $244-256$ & 1.4555 \\
\hline KMS192.1360 & $\begin{array}{l}\text { CAGCACCCAA } \\
\text { AAGGAGCCTG }\end{array}$ & $\begin{array}{l}\text { AACATTCCCA } \\
\text { GGTGCATCGC }\end{array}$ & $\begin{array}{l}\text { AGG } \\
124065\end{array}$ & $\mathrm{GCA}$ & 46 & 5 & 2 & 85 & $218-221$ & 0.3594 \\
\hline KMS202.1779 & $\begin{array}{l}\text { CGCCCTICAA } \\
\text { AAACCAAGGG }\end{array}$ & $\begin{array}{l}\text { AATTGCGACA } \\
\text { AGTCGCTCCC }\end{array}$ & $\begin{array}{l}\text { NGG } \\
144995\end{array}$ & CAG & 46 & $?$ & 5 & 100 & $257-281$ & 13841 \\
\hline RMLS222.338 & $\begin{array}{l}\text { AGACGACGAG } \\
\text { GCTTCCGATG }\end{array}$ & $\begin{array}{l}\text { GGCATAAGGT } \\
\text { TGTCGCGGAG }\end{array}$ & $\begin{array}{l}\text { NGGG } \\
009255\end{array}$ & $\mathrm{GCT}$ & 46 & 6 & 5 & 100 & $187-211$ & 0.8856 \\
\hline
\end{tabular}

* Contig is based on the M. griseea genome database,

website: http://www.genome.wi.mit.edu/annotation/fungi/magnaporthg/

\section{ACKNOWLEDGEMENTS}

We thank Miss J. Krenz, in Department of Botany and Plant Pathology, Oregon State University of USA for helpful advice and revision the paper in 
detail. This work is supported by National Basic Research Program of China (2006CB100202), Education Ministry Foundation (307025) and Doctorial Foundation of Education Ministry of China (20050676001).

\section{REFERENCES}

Benson G. 1999, Tandem repeats finder: a program to analyze DNA sequences. Nucleic Acids Res., 27: 573-580.

Dean R.A., Talbot N.J., Ebbole D.J. et al. 2005, The genome sequence of the rice blast fungus Magnaporthe grisea. Nature, 434: 980-986.

Hanks S.K. 2003, Genomic analysis of the eukaryotic protein kinase superfamily: a perspective. Genome Biol., 4, 111.

Li C.Y, Li J.B., Zhou X.G., Zhang S.S., Dong A.R., Xu M.H. 2005, Frequency and distribution of microsatellites in open reading frame of rice blast fungus, Magnaporthe grisea. Chinese J. Rice Sci., 19: 167-173. 2005. (in Chinese with English abstract)

Muller P., Weinzierl G., Brachmann A., Feldbrugge M., Kahmann R. 2003, Mating and pathogenic development of the Smut fungus Ustilago maydis are regulated by one mitogen-activated protein kinase cascade. Eukaryot. Cell, 2: 1187-1199.

Rozen S., Skaletsky H. 2000, Primer3 on the WWW for general users and for biologist programmers. Methods Mol. Biol., 132: 365-386.

Valent B. 1990, Rice blast as a model system for plant pathology. Phytopathology, 80: 33-36.

Xu J.R., Hamer J.E. 1996, MAP kinase and cAMP signaling regulate infection structure formation and pathogenic growth in the rice blast fungus Magnaporthe grisea. Genes Dev., 10: 2696-2706.

Yamada-Okabe T., Mio T., Ono N., Kashima Y., Matsui M., Arisawa M., Yamada-Okabe H. 1999, Roles of three histidine kinase genes in hyphal development and virulence of the pathogenic fungus Candida albicans. J. Bacteriol., 181: 7243-7247.

Zhang D., Yang Y., Castlebury L.A., Cerniglia C.E. 1996, A method for the large scale isolation of high transformation efficiency fungal genomic DNA. FEMS Microbiol. Lett., 145: 261265. 